\title{
Article
}

\section{SRRTC: Social Recommendation based on Relationship Transmission with Convergence Algorithm}

\author{
Huakang $\mathrm{Li}^{1,3,4}$, Yixiong Bian ${ }^{1,2}$, Xiuying $\mathrm{Xu}^{1}$ and Guozi Sun ${ }^{1,3,4 *}$ \\ 1 Jiangsu Key Lab of Big Data and Security and Intelligent Processing Nanjing University of Posts and \\ Telecommunications, Nanjing, 210023, China; huakanglee@163.com \\ 2 University of Houston 4800 Calhoun Rd. Houston, TX 77004 \\ 3 Collaborative Innovation Center for Economics crime investigation and prevention technology, Jiangxi \\ Province, China \\ 4 State Key Laboratory of Mathematical Engineering and Advanced Computing, Wuxi, 214125, China \\ * Correspondence: sun@njupt.edu.cn; Tel.: +86-189-5189-6572
}

\begin{abstract}
Social recommendation is almost as the integration of the business platform and social platform, and gradually become a top in recommendation system. Social recommendation algorithm solves the problem of cold start and data sparseness for traditional commodity, while the internal structure of the relationship graph in social relations has not been fully excavated. This paper proposes two models of Micro Relation Transfer Model and Macro Relation Transfer Model of social relations, and applies the social relations transfer models into the social recommendation system. A relationship graph is built from the relationship between customers on the Internet. Micro Relation Transfer Model establishes the transfer activation function by calculating the relationship between the two customers using the similarity of interests set. Micro Relation Transfer Model spreads the relationship of friends by calculating the proportion of common neighbors held by the customer's social relations. In order to effectively control the transmission range and effect of social relations graph, we introduce pruning algorithm based on Monte Carlo Decision Tree convergence algorithm. The experimental results show that SRRTC algorithm enhances the success rate and stability significantly.
\end{abstract}

Keywords: social recommendation; relationship graph; micro relation transfer model; macro relation transfer model; monte carlo decision tree

\section{Introduction}

With the development of cloud computing and big-data analysis, the performance of recommendation systems has been greatly developed in theory and practice. Currently, the recommendation system has become a very important technology in e-commerce and social networking, and has produced significant economic benefits. The most famous application in large and medium-sized sites, such as Facebook, Amazon, Taobao and Douban, uses various forms of recommendation algorithms to provide content, friend, and shop items recommending.

Collaborative Filtering (CF) algorithm, which is the primary applied algorithm, takes advantage of fast and accurate features. Content-based recommendation algorithm was trying to explain the interpretable relation between items in a certain extent. On the other hand, some researchers introduced the user's personal information, browsing history, and physical location information to improve the accuracy of the recommendation system. However, these methods could not solve the data sparseness and cold start problems.

Currently, some researchers use the social relations to deal with the sparseness and cold start of recommended algorithm when some online shopping or media platforms developed the social modules. According to social relations and user's feedback for relation estimation and reasoning, 
SocialTrust, such as EigenTrust [1], TidalTrust [2], MoleTrust [3], and James Caverlee
recommending theory based on the credibility of the dynamic trust reasoning model.

The existing credible recommendation methods are more concerned with dominant trust relationships, ignoring the transitivity of social relations, like: friends of friends are more likely to be friends, and birds of a feather flock together. In this paper, two trustworthiness models, Micro Relation Transfer Model and Macro Relation Transfer Model of social relations, in sociology are introduced into the social recommendation system to solve the sparseness and cold start problems. We build a relation graph of the relationship between customers on the Internet. Micro Relation Transfer Model establishes the transfer activation function by calculating the relationship between the two customers using the similarity of interest set. Micro Relation Transfer Model spreads the relationship of friends by calculating the proportion of common neighbors held by the customer's social relations. In order to effectively control the transmission range and effect of social relations graph, we introduce pruning algorithm based on Monte Carlo Decision Tree convergence algorithm. The experimental results show that SRRTC algorithm enhances the success rate and stability significantly.

This paper is organized shown as follows: Section 2 introduces the trusted recommended system and the social relation transmission research hotspot. Section 3 introduces the recommendation algorithm of friend social transfer and explains Micro and Macro's Relation Transfer Model. The optimization pruning algorithm is proposed in Section 4. Section 5 illustrates the experimental processes and measurements. The performance is discussed in Section 6. Finally Section 7 presents a summary of the work of this paper.

\section{Related Works}

The traditional recommendation systems which are mainly based on review comments collaborative filtering (CF) [5], content similarity estimation and mixed recommendation. Many algorithms in [6] have achieved good performance in e-commerce and media platforms, such as the Amazon, Taobao, and Reuters News, to solve the sparsity problems of CF.

In general, the CF method is subject to cold start and data sparsity problems. There are many literatures that propose ways to attempt to solve those problems. One possible solution is to reduce the sparsity degree by removing the non-representative user or project to narrow the user rating matrix [7]. Another approach is to identify the most appropriate user in the forecasting process by using specific methods such as specific similarity measures [8,9], pattern mining [10], social networks [11], or resource allocation [12].

Social relation (Trust relation [13]) mining or a new research hotspot that many scholars have discussed extensively in recent years. In [14], the author proposes a social network service recommendation method with trust enhancement function, called "trustman". In order to improve the accuracy of the prediction of the top k ranking, [15] proposed a method based on the objective function recommendation, in which the users are divided into two types of trustees. Based on the Tensor factorization technique, Kim and Yoon proposed a trust model in [16], which shows a trust model with additional information as a factor. In [17], the author defines the trust and reputation, and introduces the corresponding calculation method in which the trust factors have certain advantages in a film recommendation. In [2], two novel methods of using the trust network to improve the top-N recommendation are proposed. In order to alleviate the cold user problem, the author uses a special API in [3] to select the most valuable node in an aspect to calculate the probability of a similar favorite category. In [4], the author attempted to provide a personalized news recommendation in the news reading community through implicit social information to deal with cold start problems. In [18], Krauss and Arbanowski introduced the social preference ontology to solve the cold start and sparse problems.

Yilmazel and Kaleli analyzed the robustness of some typical recommended methods based on distributed data in [19]. In order to limit the behavior of malicious recommendation and fraud, Wang and Gui proposed a dynamic recommendation trust evaluation model based on 
e-commerce environment bidding in [20]. In [21], Shambour and Lu developed an implicit trust filter recommendation method and an improved user-based collaborative filtering recommendation method to select a reliable business partner to do reliable business deals. Since the trust value is calculated based on the user's average rating, the reliability and sensitivity are not high. Alejandro and Parapar used spectral clustering to deduce a clustering-based collaborative filtering algorithm in [22]. The method of sorting could accurately select the appropriate neighbors, so the performance is superior to other technologies. [23] proposed a reputation measurement method that measures service reputation to prevent malicious users from web service recommendations. By detecting malicious feedback ratings and integrating feedback anomalies, the method can identify the trustworthiness of Web services and generate prevention programs to improve the Web service recommendation performance. In [24], a multi-category recommendation system for a specific domain trust network is proposed, which used a more scientific root mean square error MAE coefficient as a measure of the recommendation system performance.

To sum up, these methods improve the recommendation system performance successfully in some degree through using trust value between users. However, social relations, as a complex social network model, have the dynamic transfer feature. Therefore, based on previous researches [25], we propose a macro friendship relation similarity expansion algorithm, and the micro-friend relation expansion algorithm of depth transfer, combined with MCTS algorithm and pruning algorithm, to optimize the collaborative recommendation system and as far as possible to solve the cold start and sparse problems.

\section{Social Relation Transmission Recommendation}

Currently, more and more social communication platforms begin to provide the online shopping model in oder to increase the incoming and user adhesion. Therefore, the social relation recommendation algorithm is also being proposed to improve the performance of traditional collaborative filtering model. In this section, the social relation transmission model will be introduced deeply after the social relation recommendation model.

\subsection{Social Relation Recommendation}

\subsubsection{Collaborative Filtering Algorithm}

Assuming there are $M$ users and $N$ items on the platform, the user set and item set can be described as User $=\left\{u_{1}, u_{2}, \ldots, u_{m}\right\}$ and Item $=\left\{\right.$ item $_{1}$, item $_{2}, \ldots$, item $\left._{n}\right\}$. We can generate a review score matrix $R_{m n}=\left\{r_{11}, \ldots, r_{i j}, \ldots r_{m n}\right\}$, if the $i^{t h}$ user buys the $j^{\text {th }}$ product, and the evaluation score for the item is $r_{i j}$. Then, we can estimate the similarity of evaluation scores between user $a$ and user $b$ using Peason model:

$$
\operatorname{sim}^{R}(a, b)=\frac{\sum_{i \subset U_{a b}} R_{a i} \times R_{b i}-\frac{\sum_{i \subset U_{a b}} R_{a i} \times \sum_{i \subset U_{a b}} R_{b i}}{n}}{\sqrt{A} \times \sqrt{B}}
$$

where

$$
\begin{aligned}
& A=\sum_{i \subset U_{a b}} R_{a i}^{2}-\frac{\left(\sum_{i \subset U_{a b}} R_{a i}\right)^{2}}{n} \\
& B=\sum_{i \subset U_{a b}} R_{b i}^{2}-\frac{\left(\sum_{i \subset U_{a b}} R_{b i}\right)^{2}}{n}
\end{aligned}
$$




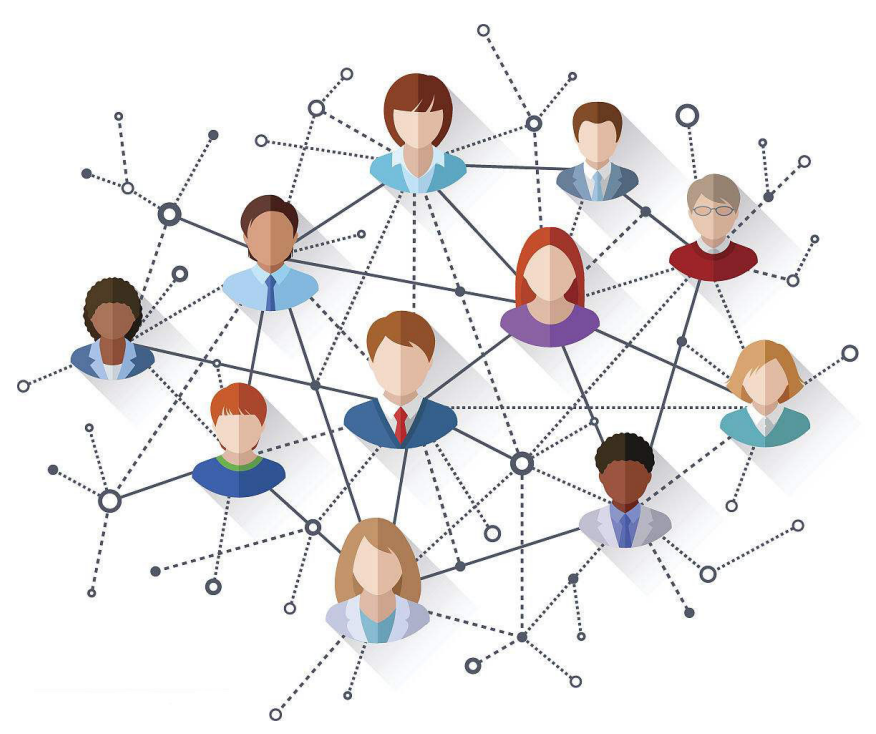

Figure 1. Social relationship network diagram

\subsubsection{Social Recommendation Algorithm}

In the social platform, we can get a friendship matrix $F=\left\{f_{11}, \ldots, f_{a b}, \ldots, f_{m m}\right\}$ where we can use $f_{a b}$ to describ friendly relationship between user $a$ and user $b . f_{a b}=1$ represents user $a$ and user $b$ is a friend, the relationship is credible, and $f_{a b}=0$ represents there is no direct relationship. Then we obtain the social relation similarity between user $a$ and user $b$ using cosine function.

$$
\operatorname{Sim}^{F}(a, b)=\frac{\sum_{a, b \in F} a b}{\sqrt{\sum_{a \in F} a^{2}} \sqrt{\sum_{b \in F} b^{2}}}
$$

Assume that the social relationship $\operatorname{Sim}^{F}$ is orthogonal to the user's interest $\operatorname{Sim}^{R}$, we can use a linear function to construct the social recommendation model $\mathrm{Sim}^{S}$ as:

$$
\operatorname{Sim}^{S}=\alpha \operatorname{Sim}^{R}+\beta \operatorname{Sim}^{F}
$$

here $\alpha$ and $\beta$ is undetermined coefficient. And the strategy of value can be described as follows:

$$
\alpha+\beta=1
$$

\subsection{Relationship Transmission Model}

In the real world, most people become friends in the following two ways (as shown in Fig. 1). On one hand, "Friends of friends are more likely to be friends", which we call it as Micro Relation Transfer Model. On the other hand, it calls "things of one kind come together, birds of a feather flock together" phenomenon. Two people have a lot of common friends, when they become friends, the surrounding friends will be more and more dense, which we defined as Macro Relation Transfer Model.

\subsubsection{Macro Relation}

If $A$ and $B$ have many common friends like $C, D$ and $E$, they are much easy to be friends. Thus, the friendly relationship transfer model is similar to width traversal, which we called Macro Relation Transfer Model. We use Pearson correlation coefficient to estimate the common friend degree 


$$
t f_{\text {macro }}(a, b)=\frac{1}{I-1} \sum_{i=1}^{I}\left(\frac{a_{i}-\bar{a}}{s_{a}}\right)\left(\frac{b_{i}-\bar{b}}{s_{b}}\right)
$$

At the same time, we assume that $a$ and $b$ can become a direct friend when the transfer relationship is larger than a threshold $\delta_{\text {macro }}$ :

$$
f^{\prime}(a, b)= \begin{cases}1 & t f_{\text {macro }}(a, b) \geq \delta_{\text {macro }} \\ 0 & \text { other }\end{cases}
$$

\subsubsection{Micro Relation}

Assuming that $A$ and $C$ are good friends of $B, B$ introduces $A$ to $C$, and $C$ introduces $A$ to his own good friend $D$. Thus, the friendly relationship transfer model is similar to deep traversal, which we called Micro Relation Transfer Model. But in real life, the trustiness between friends to pass once will be descended certainly. Therefore, we define the micro transfer model between new friendly relationship as follows:

$$
t f_{\text {micro }}(a, b)=\frac{1}{|L|} \sum_{l \in L} \frac{1}{d_{l}}
$$

where User $a$ can contact $b$ through relational path $l$ and $L$ represents all possible paths. Like the macro relation, we assume that $a$ and $b$ can become a direct friend when the transfer relationship is larger than a threshold $\delta_{\text {micro }}$ :

$$
f^{\prime}(a, b)= \begin{cases}1 & t f_{\text {micro }}(a, b) \geq \delta_{\text {micro }} \\ 0 & \text { other }\end{cases}
$$

\subsubsection{Transfor Model}

Assume that the obtained relationship matrix $T F$ can be accumulated on the original friend relationship matrix $F$ after $\mu$ intration:

$$
F^{\mu}=F^{\mu-1}+T F^{\mu-1}(a, b)
$$

\section{Transmit Optimization Method}

We believe that the transfer of friendship can improve the performance of the recommended system, but there are also some problems, such as over fitting. Therefore, we propose the following optimization methods.

\subsection{Category Recommendation}

We think that users could buy the same category product with the same functional requirements, but in the specific purchase of the product model will have their own tendencies, such as different brands and different price range. Therefore, we use the category to calculate the recommending list.

Assume that category $c_{i}$ contains $i$ product items, $\left\{\right.$ item $_{1}, \ldots$, item $\left.m_{I}\right\} \in c_{i}$, the review score of user $a$ for category is:

$$
R_{i a}^{c}=\frac{1}{I} \sum_{i=1}^{I} r_{i a}
$$

Then, the category recommendation function can be written as: 


$$
\operatorname{sim}_{c}^{R}(a, b)=\frac{\sum_{i \subset U_{a b}} R_{a i}^{c} \times R_{b i}^{c}-\frac{\sum_{i c U_{a b}} R_{a i}^{c} \times \sum_{i \subset U_{a b}} R_{b i}^{c}}{n}}{\sqrt{A} \times \sqrt{B}}
$$

\subsection{Optimization Methods}

We propose the MCST Direction Optimization and Threshold Pruning Optimization methods to deal with the credibility problem in the relationship transfer.

\subsubsection{MCST Direction Optimization}

In the real society, the friendship between people will decrease with the increase of the transmission number of layers. Therefore, we introduce the MCTS algorithm to guide the Macro and Micro relations. MCTS algorithm is divided into three parts: selection, transmission, backtracking. The details are as follows:

Select: From the root node ( $A$ layer node), recursively select the $B$ layer node $L$ that meets the following convergence constraints.

$$
\begin{array}{r}
\Delta(n)=F^{n}-F^{n-1} \\
\lim _{n \rightarrow \infty} \Delta(n)<\gamma
\end{array}
$$

$F^{n}$ is the $n$th transfer. The transmission will be terminated when the difference between two transfer results is less than the threshold $\gamma$.

Transmission: If it does not terminate at L-level, it will continue to look for its child nodes (C-level nodes), traverse its child nodes ( $L_{i}$ in the $C$ layer) and add the above convergence constraints again at $L^{\prime}$.

Backtracking: Update the value on the current eligible users $_{i}$ action sequence (create the relationship between layer $A$ and layer $C$ ).

\subsubsection{Threshold Pruning Optimization}

In this section, we use the threshold pruning algorithm to preprocess the similarity matrix of the original user, similarity matrix of the original friedn relationship, similarity matrix of the Macro transfer relation in expansion and similarity of the Macro transfer relation in expansion, processing method is as follows:

$$
S_{c u t}=\sum_{i=0}^{n} \operatorname{Cut}\left(S_{i} \leq \delta\right)
$$

$S_{i}$ is a certain similarity in the matrix of similarity needed to be processed, and $\delta$ is a threshold. If similarity between two users is less than the threshold in the similarity matrix, this similarity between the users will be deleted.

After the data is preprocessed by the pruning algorithm, the friend relations with weak reliability in the original data are removed, and a new similarity $S_{c u t}$ matrix could be obtained. We could finish the recommendation based on the cut similarity $S_{c u t}$.

\section{Experimental Environments}

\subsection{Experimental Data and Environment}

The data set used in this paper is from the prestigious e-commerce commodity evaluation website Epinions.com. The amount of data is shown in Table 1. The user scores to the item are from 1 to 5 in 
Table 1. Experimental dataset size

\begin{tabular}{cc}
\hline Table Name & Data Size \\
\hline User & 131228 \\
Product & 317755 \\
Category & 587 \\
Review & 1127673 \\
Similarity & 3689606 \\
Trust & 538391 \\
\hline
\end{tabular}

Review table, and the trust defaults are 1.0. Through calculation and analysis, we find that there are 17599 users who do not exist the score information, more than half of the user scores are less than 5 or the number of direct trusts is less than 5 which means the data set is very sparse.

\subsection{Measure of Recommended Algorithm}

1) Recommended Success Rate (SR)

$$
S R=\frac{\sum_{R \in R_{\text {test }}}\left(P_{R} \cap \sum_{R^{\prime} \in R_{\text {top } N-S}} P_{R^{\prime}}\right)}{R_{\text {test }}}
$$

$R$ is the user needed to be recommended. $R^{\prime}$ is the users whose similarity with the user $R$ ranks among Top $-N$ in the corresponding data table. $R_{\text {test }}$ is the test user set. $P_{R}$ is the item number (category) set purchased by $R$. $P_{R}$ ' is the number (category) set of items purchased by the Top- $N$ users. $R_{t o p N-S}$ represents the Top $-N$ users set with the highest similarity to the user $R$ in the data table $S$.

In the experiment, the recommended success rate is used to measure the macro-quality of each recommendation algorithm. The higher $S R$, the better recommendation performance.

$$
M A E=\frac{\sum R_{u, i \in R_{\text {test }}}\left|R_{u, i}-R_{u, i}^{\prime}\right|}{R_{\text {test }}}
$$

$\left|R_{\text {test }}\right|$ is the number of reviews in Review table. $R_{u, i}$ and $R_{u, i}^{\prime}$ respectively represent the amount of user's actual comment (purchase) and user's predicted comment (purchase). Particularly, $\left|R_{u, i}-R_{u, i}^{\prime}\right|$ is Boolean minus.

Since MAE is absolutized, there is no case of positive and negative to be offset, which could better reflect the actual situation of the prediction error. Therefore, we use MAE to determine the recommended system micro-quality level. The smaller MAE value, the lower error of the recommended algorithm, the more stable recommendation, and the higher performance.

\section{Results}

In the experiment, we use four methods, traditional Collaborative Filtering (cf), social-based recommendation (friend), Macro transfor recommendation (Macro) and Micro transfor recommendation (Micro) for results comparison. 


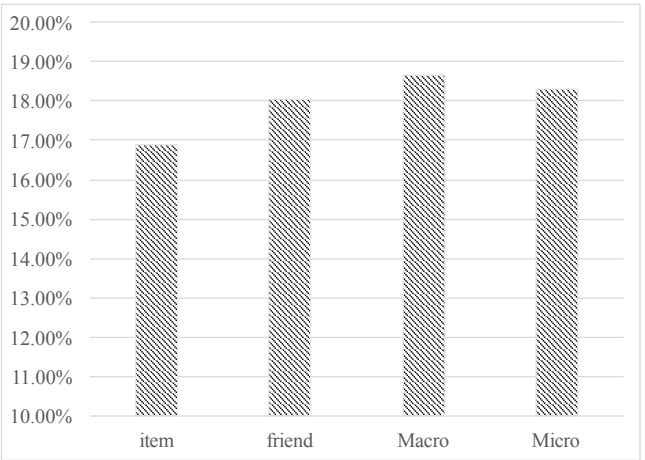

(a) Results of SR

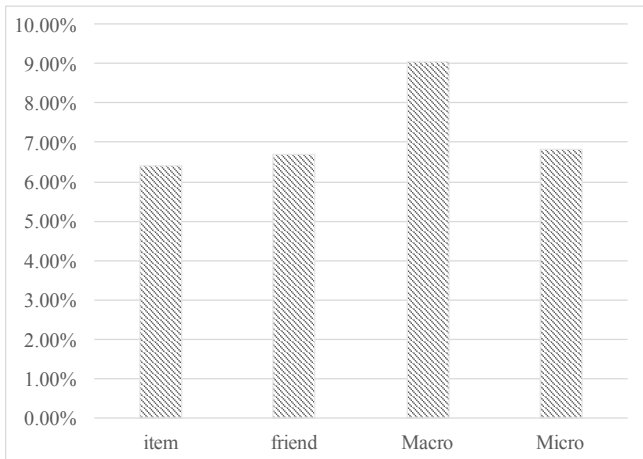

(b) Results of MAE

Figure 2. Collation Map Under Different Recommendation

Figure 2 shows the the SR and MAE results on the category recommendation. We can see that the Macro and Micro algorithms are improved by $3.4 \%$ and $1.5 \%$, respectively compared with existing friend social-based collaborative recommendation algorithm in Figure 2 (a). However, the MAE for the Macro and Micro algorithms alos increased for Macro and Micro algorithms, which means that this two kinds of extended algorithms have decreased in the recommended stability in in Figure 2 (b). The Macro and Micro delivery have some improvement on the accuracy of the recommendation.

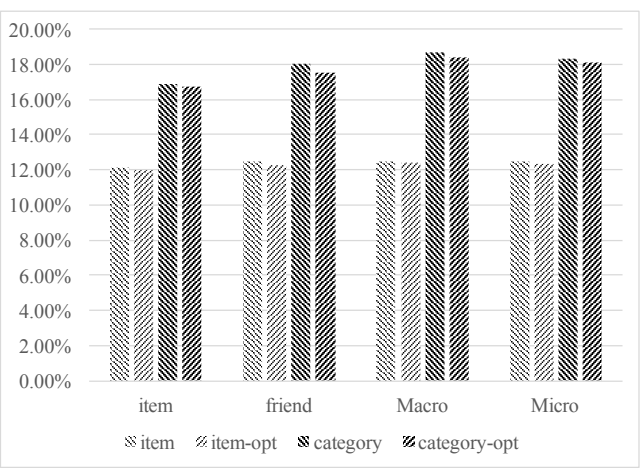

(a) Results of SRs

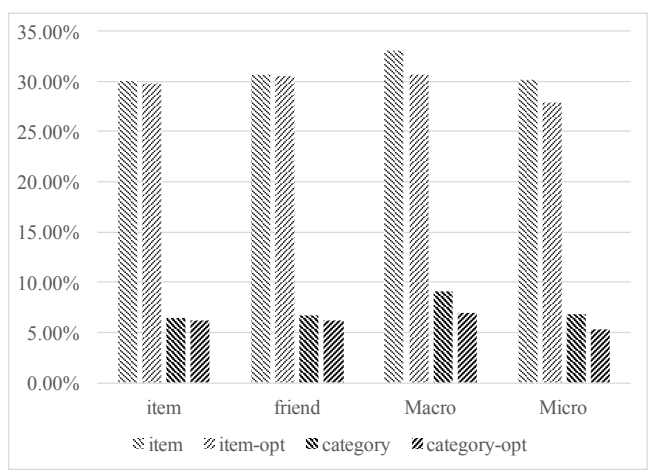

(b) Results of MAEs

Figure 3. Collation Map Under Different Optimization

In order to analyze the experimental results more clearly, we show the effect of item-based and category-based recommendations with different methods in Figure 3. Figure 3 (a) illustrates the SR results for item-based and category-based with four recommendation methods. We can clearly see that the SRs results based on category recommendations are almost more than $4 \sim 5 \%$ in average comparing with the item-based SRs. Moreover, the proposed optimization pruning algorithm basically does not reduce the SR results of each method. Figure 3 (b) illustrates the MAE results for item-based and category-based with four recommendation methods. It is very clear that the MAE value based on category recommendations is much smaller than the item-based recommendation results. The proposed pruning algorithms can effectively suppress the recommended error conditions for Macro and Micro transfor.

\section{Conclusions}

This paper proposed relations transmission in the social recommendation system based on the social relation theory of internal structure of relation graph. We named the deep spread of relation as Micro Relation Transfer Model while the wide spread of relation was Macro Relation Transfer 
Model. Micro Relation Transfer Model is considering that birds of a feather flock together, transformed the relation with maximum interest similarity. Macro Relation Transfer Model is conceding that people who have mutual friends are most likely to friends, spread the relationship by the proportion of common neighbors. The Monte Carlo Decision Tree convergence algorithm was introduced to diminish the effect of social transmission. We used epinion dataset to enhance the performance of the entire recommended system successfully.

Acknowledgments: This work was supported by the NSFC (No.61502247, 11501302, 61502243, 91646116), China Postdoctoral Science Foundation (No.2016M600434), Natural Science Foundation of Jiangsu Province (BK20140895, BK20150862), Scientific and Technological Support Project (Society) of Jiangsu Province (No.BE2016776), and Postdoctoral Science Foundation of Jiangsu Province(1601128B), Opening Project of Collaborative Innovation Center for Economics crime investigation and prevention technology(JXJZXTCX-015), and Open Project Program of the State Key Laboratory of Mathematical Engineering and Advanced Computing(2017A10).

\section{References}

1. M. Morita , Y. Shinoda, in: B. Croft, C.J. van Rijsbergen (Eds.), Information Filtering Based on User Behavior Analysis and Best Match Text Retrieval, 1994, pp. 272-281.

2. G. Guo , J. Zhang, N. Yorke-Smith , Leveraging multiviews of trust and similarity to enhance clustering-based recommender systems, Know. Based Syst. 74 (2015) 14-27.

3. M. Ramezani , P. Moradi , F. Akhlaghian, A pattern mining approach to enhance the accuracy of collaborative ?ltering in sparse data domains, Physica A 408 (2014) 72-84.

4. A. Javari , M. Izadi , M. Jalili , Recommender systems for social networks analysis and mining: precision versus diversity, Complex Syst. Netw. (2015) 423-438.

5. Schafer J B, Dan F, Herlocker J, et al. Collaborative Filtering Recommender Systems[J]. Acm Transactions on Information Systems, 2004, 22(1):5-53.

6. Hosale N S, Pande S S. A Survey on Recommendation System for Big Data Applications[J]. Journal of Biological Engineering, 2015, 7(1):67-73.

7. H. Chen, S. LV. Study on trust-based collaborative filtering recommendation strategy. Journal of Computational Information Systems, Binary Information Press, 2012, 8(10): 4095-4100.

8. D. Sepandar, T. Mario, The Eigentrust algorithm for reputation management in p2p networks. Proceedings of the 12th international conference on World Wide Web, Budapest, HUNGARY, 2003: 640-651.

9. J. Ann, Golbeck. Computing and applying trust in web-based social networks. PhD thesis, University of Maryland, 2005.

10. P. Massa, P. Avesani. Trust Metrics on Controversial Users: Balancing Between Tyranny of the Majority and Echo Chambers. International Journal on Semantic Web and Information Systems, 2007, 3(1): 39-64.

11. J. Caverlee, L. Liu, S. Webb. Towards Robust Trust Establishment in Web-Based Social Networks with SocialTrust. Proceedings of the 17th international conference on World Wide Web, Beijing, China,2008: 1163-1164.

12. M. Papagelis, D. Plexousakis, T. Kutsuras. Alleviating the Sparsity Problem of Collaborative Filtering Using Trust Inferences. Proceedings of the 3rd International Conference on Trust Management (iTrust-05). Springer Berlin Heidelberg, 2005: 224-239.

13. A. Josang, S. Marsh, S. Pope. Exploring Different Types of Trust Propagation. Proceedings of the 4th International Conference (iTrust-06), Pisa, Italy. Springer Berlin Heidelberg, 2006: 179-192.

14. Z. Sai, Commodity recommendation system based on social network trust model. Guangzhou: South China University Of Technology, 2012.

15. B. Zhang, Z. Huang, J. Yu, Y. Xiang. Trust Computation for Multiple Routes Recommendation in Social Network Sites. Security and Communication Networks, 2014. DOI: DOI:10.1002/sec.935.

16. J. Lu , D. Wu , M. Mao , W. Wang , G. Zhang, Recommender system application developments: a survey, Decis. Support Syst. 74(2015) 12-32.

17. B. Sarwar , G. Karypis, J. Konstan, J. Riedl , Item-based collaborative ?ltering recommendation algorithms, in: Proceedings of the 10th International Conference on World Wide Web, Publishing, Hong Kong, 2001, pp. 285-295.

18. A. Javari , J. Gharibshah , M. Jalili, Recommender systems based on collaborative ?ltering and resource allocation, Soc. Netw.Anal. Mining 4 (2014) 1-11. 
19. P. Moradi, S. Ahmadian, F. Akhlaghian, An effective trust-based recommendation method using a novel graph clustering algorithm, Physical436 (2015) 462-481.

20. S. Deng, L. Huang, G. Xu, Social network-based service recommendation with trust enhancement. Expert Syst. Appl. 2014, 41,8075-8084.

21. Can, Bharat, B. Sort: A self-organizing trust model for peer-to-peer systems. IEEE Trans. Dependable Secur. Comput. 2013, 10, 14-27.

22. C. Park, D. Kim, J. Oh, H. Yu, Improving top-K recommendation with truster and trustee relationship in user trust network. Inf. Sci. 2016, 374, 100-114.

23. S. Kim, Y. Yoon, Recommendation system for sharing economy based on multidimensional trust model. Multimed. Tools Appl. 2016, 75, 15297-15310.

24. M. Ozsoy, F. Polat, Trust based recommendation systems. In Proceedings of the 2013 IEEE/ACM International Conference on Advances in Social Networks Analysis and Mining, Niagara Falls, ON, Canada, 25-28 August 2013; pp. 1267-1274.

25. A Josang, S Marsh, S Pope. Exploring Different Types of Trust Propagation. Proceedings of the 4th International Conference (iTrust-06), P-isa, Italy. Springer Berlin Heidelberg, 2006: 179-192. 